\title{
All-Printed Piezoresistive Sensor Matrix with Organic Thin-Film Transistors as a Switch for Crosstalk Reduction
}

V. Correia ${ }^{\mathrm{A}}$, J. Oliveira ${ }^{\mathrm{A}, \mathrm{B}}$, N. Perinka ${ }^{\mathrm{C}}$, P. Costa $^{\mathrm{B},{ }^{*}}$, E. Sowade ${ }^{\mathrm{D}, \mathrm{E}}$, Kalyan Y. Mitra ${ }^{\mathrm{D}, \mathrm{F}}$, R.R. Baumann ${ }^{\text {D,F }}$, S. Lanceros-Mendez ${ }^{\mathrm{C}, \mathrm{G}}$,*

A. Dr. V. Correia, Dr. J. Oliveira

Algoritmi Research Center, University of Minho, Campus de Azurém, 4800-058 Guimarães, Portugal

B. Dr. J. Oliveira, Dr. P. Costa

Centro de Física, University of Minho, 4710-057, Braga, Portugal

E-mail: pcosta@fisica.uminho.pt

C. Dr. N. Perinka, Prof. S. Lanceros-Mendez

BCMaterials, Basque Center for Materials, Applications and Nanostructures, UPV/EHU Science Park, 48940 Leioa, Spain

E-mail: senentxu.lanceros@bcmaterials.net

D. Prof. E. Sowade, Prof. Kalyan Y. Mitra, Prof. R.R. Baumann

Digital Printing and Imaging Technology, Technische Universität Chemnitz, 09107, Chemnitz, Germany

E. Prof. E. Sowade

Zschimmer \& Schwarz Mohsdorf GmbH \& Co. KG, Textile Auxiliaries Division, 09217, Burgstädt, Germany

F. Prof. Kalyan Y. Mitra, Prof. R.R. Baumann

Fraunhofer Institute for Electronic Nano Systems, Department Printed Functionalities, 09126, Chemnitz, Germany

G. Prof. S. Lanceros-Mendez

IKERBASQUE, Basque Foundation for Science, 48013 Bilbao, Spain

Keywords: Printed electronics, OTFTs, piezoresistive, strain sensor, carbon nanotubes

\begin{abstract}
A generation of piezoresistive sensors (force or deformation) fully processed by printing technologies is increasingly being implemented in applications due to advantages as large-area application, simple device integration and high flexibility.

This work reports the development of a fully printed piezoresistive ( $5 \times 5$ sensor) matrix in which an organic thin-film transistor (OTFT) is placed in each sensor in order to allow the readout of each sensor independently and thus reducing the crosstalk between individual sensors. The manufacturing was carried out using inkjet printing for the deposition of materials in a thin layer stacked on top of each other to obtain functional OTFTs. Screen-printing was applied for the deposition of piezoresistive nanocomposite layer based on multi-walled carbon nanotubes within an elastomeric styrene-ethylene-
\end{abstract}


butadiene-styrene (SEBS) polymer matrix. The fabrication and characterization of both OTFT and sensors are presented and discussed in detail. The inkjet-printed OTFTs with a width/length ratio of the channel about 130 showed a drain-source current (IDS) of 150 $\mu \mathrm{A}$ with a gate-source voltage of $-40 \mathrm{~V}$. With respect to the sensors, gauge factors up to 5.9 were obtained, resulting in a current variation of $1.5 \mu \mathrm{A}$. This corresponds to about $0.7 \%$ of the total IDS in a deformation cycle.

\section{Introduction}

There are an increasing interest and demand for sensors based on the continuous growth of smart and portable electronic devices and the development of the internet of things (IoT) system ${ }^{1}$. Among the most demanded sensors are temperature, humidity, pressure ${ }^{2,3}$ and accelerometers for physical sensing ${ }^{4-7}$, biosensors ${ }^{2,5,8}$, and gas chemical sensors ${ }^{9,10}$. Most of this demand will be met by the exploitation of silicon technology, mainly in the fields of computing, communication and data processing ${ }^{11-13}$ that have comparable highperformance requirements. But in many other applications, characterized primarily by the need of large-area sensing, implementation of large number of sensors, low-cost production or mechanical flexibility of the sensors, printed sensors represent a needed and suitable solution ${ }^{3,14}$.

In recent years, printed electronics has taken important steps in its consolidation as an alternative technology for the fabrication of organic thin-film transistors (OTFTs) ${ }^{15,16}$, displays $^{17}$, sensors ${ }^{18-20}$, electronic circuits $^{5,21,22}$, and batteries ${ }^{23,24}$, among others.

This has been achieved by unique advantages of printing electronics over using traditional silicon technology, such as physical flexibility of the devices, low-cost processing, environmental friendliness, potential biocompatibility, transparency of printed layers and fabrication over large areas on temperature-sensitive materials ${ }^{14,25}$. 
In particular, the area of piezoresistive printed sensors is one of the fields in which relevant developments have been observed, being also still one of the fields with to larger perspective of growth and applicability ${ }^{26}$. These developments are typically based on piezoresistive composites, combining easy processing and low effective cost fabrication with high flexibility and conformability to surfaces, low power consumption, and the use of simple readout electronics ${ }^{27,28}$.

While the main fabrication method of commercially available piezoresistive matrix sensor is the conventional CMOS technology or highly specialized vacuum deposition methods, it is reported ${ }^{29-31}$ that printed sensors are still at their early stages and, in most cases, being demonstrated using hybrid processing techniques, involving some phases of the process through printing technologies and the remaining phases using conventional methods $^{32,33}$. Carbon nanotubes (CNT) and CNT polymer composites have been successfully used in flexible or strain sensing, but with limitations in terms of stretchability and repeatability ${ }^{34-37}$. On the other hand, with respect to sensors in a matrix format, the difficulty in the reading process is higher due to the effect of cross-talking between the various sensors ${ }^{38}$. This effect can be attenuated through the intensive processing of the obtained signal ${ }^{38,39}$. However, for cases of multiple sensor actuation, the intensive process of the signals becomes quite complicated and prone to errors. In this context, the present work introduces a fully printed piezoresistive sensors matrix with an OTFT associated with each sensor. The OTFTs reduce the cross-talking effect between sensors and are produced by inkjet printing.

Among the different printing technologies used for the area of printed electronics, inkjet printing has been gaining increasing attraction ${ }^{40,41}$ due to the inherent advantages of noncontact, high precision and mask-less additive patterning. 


\section{Experimental details}

\subsection{Materials and their preparation for the fabrication of the printed OTFT matrix}

Polyethylene naphthalate (PEN) films (Dupont Teijin Q65FA) with a thickness of 125 $\mu \mathrm{m}$ were employed as flexible polymeric substrates. Before starting the printing process, the substrate was cleaned using ethanol and subsequently dried using a laminar flow of nitrogen, avoiding the existence of particles on the surface. UTDAgIJ1 conductive silver nanoparticle ink from UT DOTS (UT Dots Inc., USA) was used for the manufacturing of the electrodes. The ink was filtered through a $0.2 \mu \mathrm{m}$ syringe filter before printing. The applied dielectric material was cross-linked poly-4-vinylphenol (c-PVP, purchased from Sigma Aldrich with a molecular weight of about 25 000). PVP was dissolved at room temperature in $10 \mathrm{~mL}$ propylene glycol monomethyl ether acetate (PGMEA), and magnetically stirred for $3 \mathrm{~h}$. Poly(melamine-coformaldehyde) methylated (PMFM, from Sigma Aldrich, molecular weight about 432, 84 wt.\% in 1-butanol) was added as a crosslinking agent under stirring for $2 \mathrm{~h}$. The ratio of PVP to PMFM was 2:1. Before printing, the dielectric ink was filtered through a $0.2 \mu \mathrm{m}$ syringe filter to ensure the removal of all residual agglomerations. Poly(3-hexylthiophene-2,5-diyl) (P3HT):phenyl-C61-ButyricAcid-Methyl Ester (PCBM), formulated as inkjet ink (Plexcore PV 1000) was used as heterojunction $\mathrm{p}-\mathrm{n}$ organic semiconductor (OSC) material for the fabrication of the bottom-gate top-contact OTFTs.

It is interesting to notice that being printed electronics a very recent area, under development, there are still few commercial functional inks with good and reproducible performance for TFT at low prices, compatible with industrialization. Semiconductor inks are among the most relevant ones for many applications, being inks with a suitable 
performance for TFT, such as PCBM, and competitive prices developed in areas such as printed solar cells ${ }^{38}$, where large volumes of semiconductor inks are needed.

\subsection{Fabrication of the OTFT matrix}

Inkjet printing was performed with a Dimatix Materials Printer (DMP) 2831 (Fujifilm Dimatix Inc., USA), equipped with 16 nozzles piezoelectric printhead with a nominal drop volume of about $10 \mathrm{pL}$ and a nozzle-to-nozzle spacing of $254 \mu \mathrm{m}$. For all inks, the printing process was carried out with a maximum jetting frequency of $5 \mathrm{kHz}$ and a maximum drop ejection voltage of $35 \mathrm{~V}$.

Figure 1 shows the waveform used in the printing process for each type of ink. The waveforms were explicitly obtained after a process of experimental analysis and optimization of the formation of the ejected inkjet droplets, as well as the analysis and optimization of the print resolution for each of the inks ${ }^{42}$.

The ink is ejected from the nozzle, through the excitation of the piezoelectric printhead, using the feature of the dropwatcher in the printer, it was possible to evaluate the result of the optimizations carried out in the excitation signal. Generally, it starts with a standard waveform provided by the equipment, or in some cases, by the ink supplier. Then, the optimization process can be decomposed into three phases. It starts by optimizing pulse width, which corresponds to the factor with the largest influence on the droplet formation, varying droplet volume, velocity and formation of satellite drops at the same time.

The second phase corresponds to the voltage optimization, where the objective is to obtain the highest possible velocity that produces a reliable droplet without tail potentially breaking up into satellites. Finally, the jetting frequency adjustment is carried out, which allows accelerating the printing process, which is particularly relevant for industrial 
production and large format printing, leading to significant savings of the printing time, always ensuring that there is no deterioration of the characteristics of the formed drops.

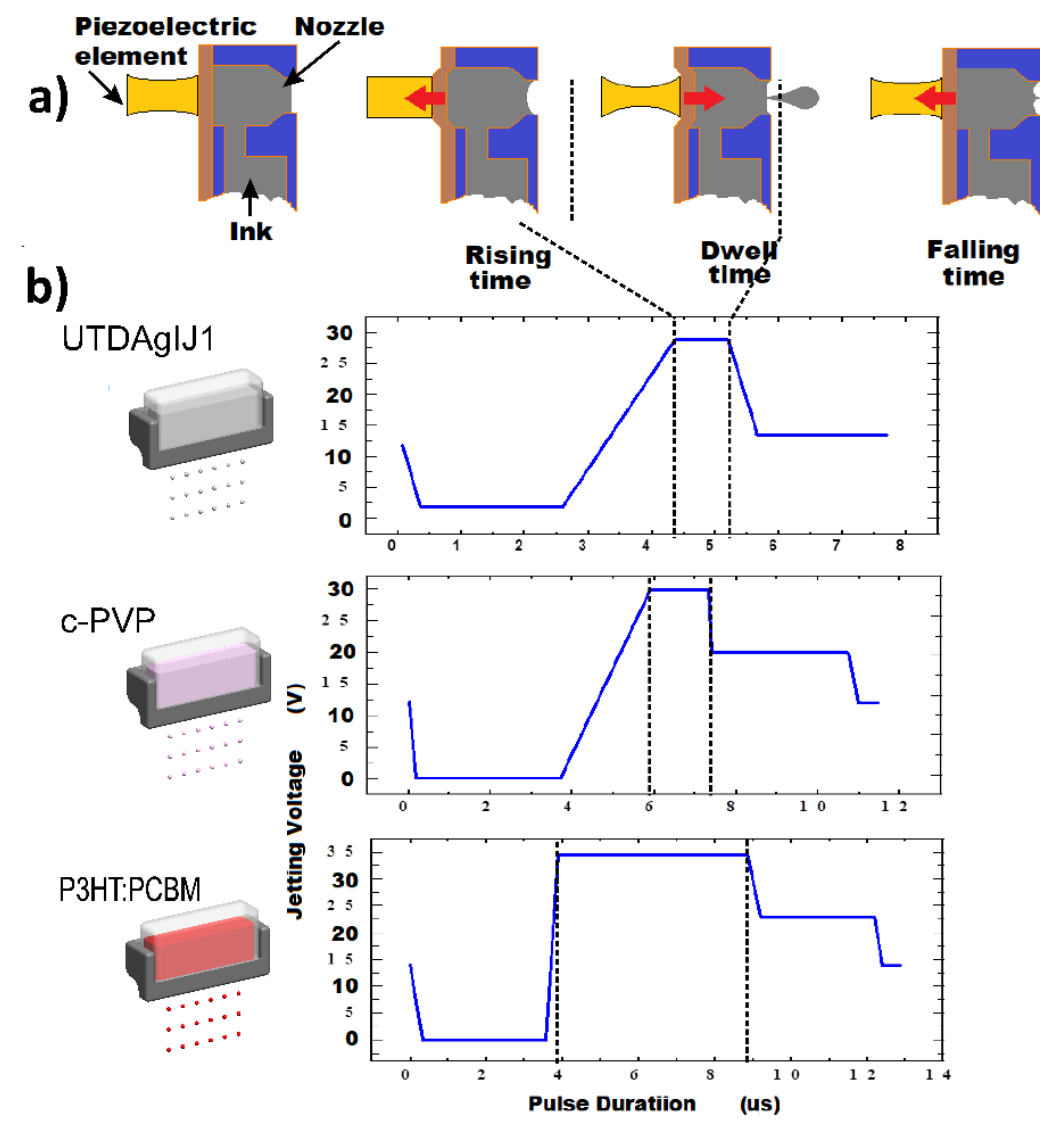

Figure 1. Schematic representation of the printhead piezoelectric mechanism a), and jetting waveform for the silver, dielectric and OSC inks b).

The waveforms are characterized by a relatively long rising time, in the order of $4 \mu \mathrm{s}$, allowing to increase the volume of the ejected droplet. The dwell time has been set according to the characteristics of the ink, in the order of $1 \mu$ s for the conductive and dielectric ink, and in the order of $5 \mu$ s for the OSC ink. Further, it was verified that for the elimination of satellite drops in the OSC and dielectrics inks, an intermediate stage in the falling time segment was required, which supported the pinch-off of the droplet.

Figure 2 shows the bottom-gate top-contact OTFT architecture for the development of the $5 \times 5$ piezoresistive sensing matrix to measure, independently, a large number of 
sensor's per unit of area for accurate determination of the zone subjected to an external stimulus. Each OTFT consists of four different layers: (I) a rectangular bottom gate layer with contact pads fabricated using the silver nanoparticle ink, (II) a rectangular insulating dielectric layer based on c-PVP, (III) 3 inter-digitated source-drain (S-D) electrodes using the silver ink, and (IV) an OSC layer on top of the stack at the area of the S-D channel. The (V) piezoresistive layer is printed on top of the OTFTs by screen printing to achieve high-performance thick layers ${ }^{43,44}$.

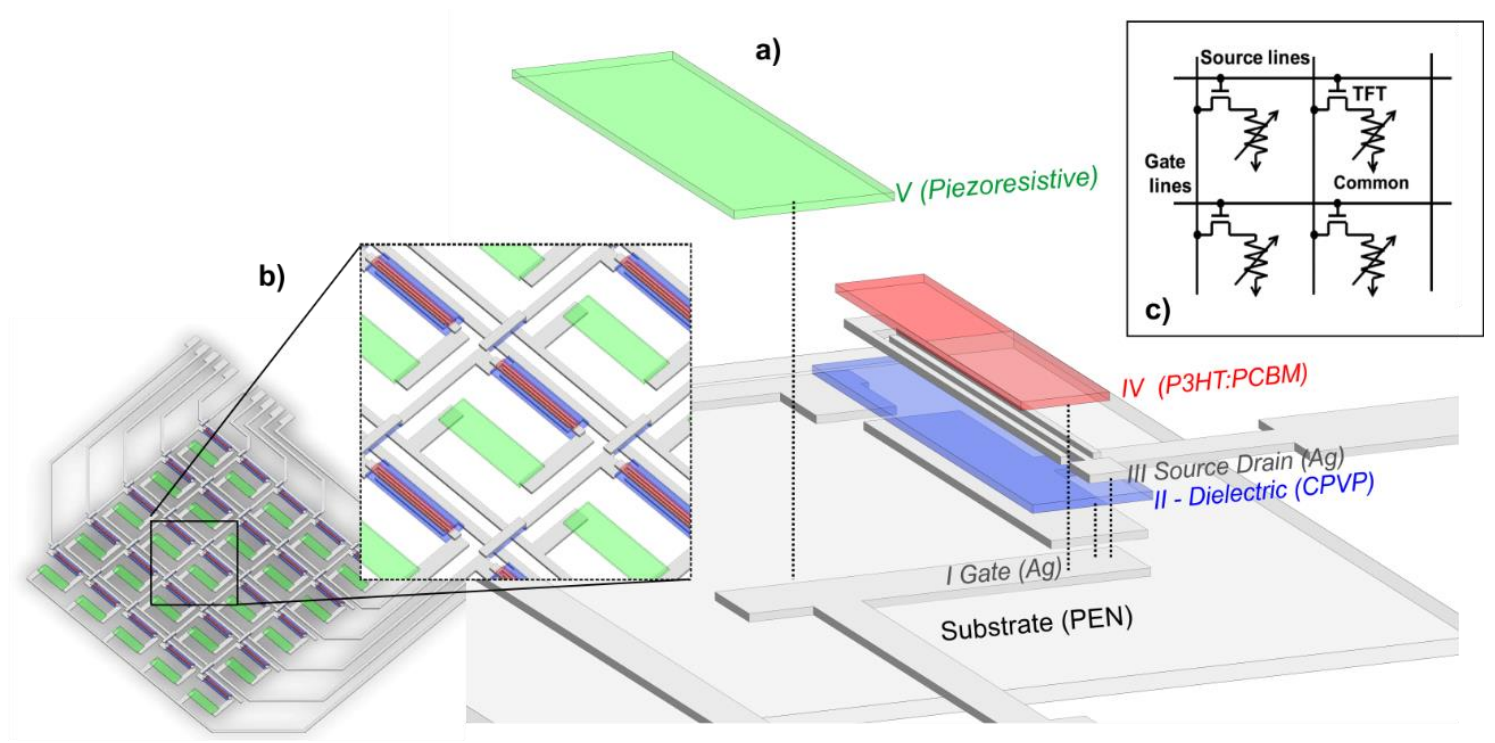

Figure 2. a) Schematic representation of the OTFT-based piezoresistive device structure; b) layout of the inkjet-printed $5 \times 5$ piezoresistive sensors; c) electric model of the piezoresistive matrix.

The spacing between source and drain electrode fingers in the digital design was $80 \mu \mathrm{m}$ and represents the channel length of the OTFTs. The channel width in the digital design is $10.4 \mathrm{~mm}$.

For the printing of the first gate electrode layer (using silver ink), a resolution of $635 \mathrm{dpi}$ was adopted, corresponding to a distance between the deposited droplets of $40 \mu \mathrm{m}$. During this phase of the process, the printhead was maintained at $35{ }^{\circ} \mathrm{C}$ and the temperature at the substrate was set at $40{ }^{\circ} \mathrm{C}$. After the layer has been printed, it was 
subjected to the drying and sintering process in a convection oven (JP Selecta 2005164) at $150^{\circ} \mathrm{C}$ for $30 \mathrm{~min}$, as indicated in Figure 3 a- process 1 ).

Then the c-PVP layer was printed. Given the characteristics of the ink and the need to ensure that there was no short circuit between the layers, a resolution of $1270 \mathrm{dpi}$ corresponding to a distance between the droplets of $20 \mu \mathrm{m}$ was used. This ink requires no heating during processing and the substrate was maintained at room temperature $(\approx 28$ ${ }^{\circ} \mathrm{C}$ ). Once printed, the curing process was performed at a temperature of $150{ }^{\circ} \mathrm{C}$ for 30 $\min$.

For the S-D electrodes, the same printing and curing characteristics previously mentioned for the first layer were applied.

The final layer for the OTFTs stack was the active OSC material, (P3HT:PCBM) that was printed with a drop space of $20 \mu \mathrm{m}, 35^{\circ} \mathrm{C}$ substrate temperature, $28{ }^{\circ} \mathrm{C}$ printhead temperature and curing temperature of $100{ }^{\circ} \mathrm{C}$ for $10 \mathrm{~min}$ in an oven. During the printing process, a quality check of the printed patterns was performed using a Leica DM4000 M microscope. 


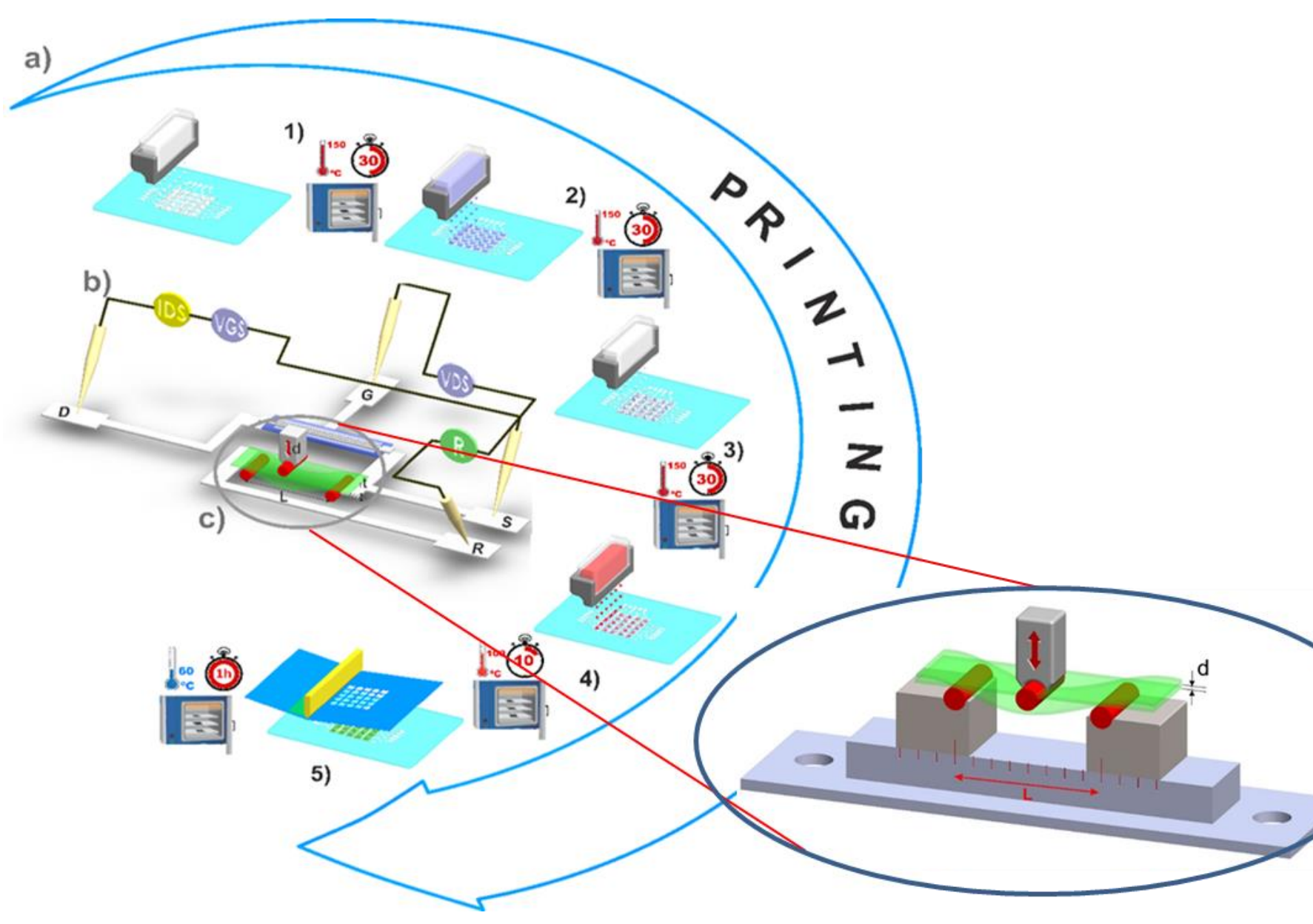

Figure 3. Schematic representation of the fabrication process for the OTFTs and the corresponding curing conditions a): inkjet printing of 1) silver bottom layer; 2) dielectric layer of c-PVP ink; 3) silver layer corresponding to the drain and source electrodes; 4) active semiconducting material OTFT layer (P3HT:PCBM) and 5) screen-printing of the piezoresistive ink layer, b) Schematic representation of the electrical and functional characterization setup of the transistor-based piezoresistive sensors. The OTFT characterization parameters, drain-source voltage (VDS) and drain-source current (IDS) were measured for different gate-source voltages (VGS), using the terminals drain (D), source $(\mathrm{S})$ and gate $(\mathrm{G})$; c) schematic representation of the experimental 3-point bending mechanical test procedure with the final device and magnification of the 3-point mechanical bending set up. 


\subsection{Development and application of piezoresistive ink layer}

The piezoresistive inks for screen printing were prepared by using $1 \mathrm{~g}$ of styreneethylene/butylene-styrene (SEBS) in $6 \mathrm{~mL}$ of cyclopentyl methyl ether (CPME), leading to a viscosity of about 1400 and $2500 \mathrm{cP}$, evaluated with a Physica MCR 300 Modular Compact Rheometer at shear rates between 0 and $1600 \mathrm{~s}^{-1}$. The ink was prepared as following (general guidelines are presented $\mathrm{in}^{43,45}$ ): 4 weight percentage (wt.\%) of multiwalled carbon nanotubes (MWCNT, Nanocyl, reference NC700), diameter of $9.5 \mathrm{~nm}$ and length of $1.5 \mu \mathrm{m}$ ) was placed in an Erlenmeyer with the corresponding amount of toluene and placed in an ultrasonic bath (Sonorex Super - RK 106) for approximately $6 \mathrm{~h}$, to avoid agglomeration and obtain good MWCNT dispersion. After this step, SEBS was added to the solution and placed under magnetic stirring until complete dissolution of the polymer.

After ink preparation, the material was printed with a semi-automatic screen printer model DX-3050D (DSTAR) with a metallic vacuum table (Figure 3a- process 5). A polyester mesh screen was used within an aluminum frame $\left(450 \times 450 \mathrm{~mm}^{2}\right)$ and a profile of $20 \times 20 \mathrm{~mm}^{2}$. The printing screen mesh is characterized by 100 threads/cm with a thread diameter of $40 \mu \mathrm{m}$ and a square-edged mesh opening of $56 \mu \mathrm{m}$. The squeegee orientation angle was set to $45^{\circ}$ relative to the printing substrate and the tension on the mesh was 20 N. Once printed, the sensors were placed in an oven (Binder E, model 28) at a temperature of $60{ }^{\circ} \mathrm{C}$ during $1 \mathrm{~h}$ for complete solvent evaporation. The final thickness of the piezoresistive layer was around $15 \mu \mathrm{m}$. More details on the processing of piezoresistive inks can be found in ${ }^{43}$.

\subsection{Characterization of the printed sensor matrix}


Scanning electron microscopy (SEM) images were obtained with a Zeiss Auriga microscope. The system is equipped with a focused ion beam (FIB) tool Zeiss 1560XB Cross Beam. FIB cuts were performed to obtain cross-sectional images of the layer stack in order to determine the thickness and the structure of the individual layers.

The electrical characterization of the printed matrix is the same as the electrical characterization of an OTFT. According to the schematic representation in Figure $3 \mathrm{~b}$, first, the IDS was measured in relation to the VDS in the range of 0 to $-30 \mathrm{~V}$, for the VGS ranging from $-40 \mathrm{~V}$ to $0 \mathrm{~V}$ in steps of $-10 \mathrm{~V}$. Thus, the voltage needed for the OTFT to be operating in the saturation region is obtained.

In order to ensure the reproducibility of the results, the functionality of all printed OTFTs within the array was analyzed. The functional performance of the printed OTFT matrix with and without the printed piezoresistive layer was further measured through electrical tests. The electrical measurements of the OTFT were performed with a QuadTech 1920 Precision LCR Meter. The electrical operation properties of the printed piezoresistive sensor were characterized with a 2400 source/meter and a 6457 picoammeter with integrated voltage source from Keithley (Keithley Instruments, Cleveland, OH, USA) controlled by a labVIEW program developed for current vs. voltage (I-V) characteristic measurements. The probe station was directly connected to the piezoresistive sensor using gold-coated round tips. The IEEE1620 standard for test methods for the characterization of OTFTs and materials was taken into consideration during the measurement.

Piezoresistive response tests were carried out in bending mode by measuring simultaneously the loading-unloading mechanical and electrical response of the materials. Three-point-bending mechanical tests (schematically represented in Figure 3c), were performed in a Shimadzu AG-IS universal test set-up by applying four cycles at different deformations up to $0.1 \mathrm{~mm}$, while the electrical resistance was simultaneously recorded 
with an Agilent 34410A multimeter, through silver electrodes placed on the piezoresistive sensor (Figure 3c).

The variation of the electrical resistance under mechanical solicitation and the corresponding piezoresistive sensibility were calculated for each loading-unloading cycle and provided as the value of the piezoresistive sensibility. For the three-point-bending tests, four load-unload cycles were performed up to $0.1 \mathrm{~mm}$ of displacement at a test velocity of $10 \mathrm{~mm} / \mathrm{min}$. The sample bending in the three-point-bending measurements was calculated from the theory of pure bending of a plate to a cylindrical surface, using equation 1.

$$
\varepsilon=6 \frac{t z}{L^{2}}
$$

where $z$ is the vertical displacement of the piston, $t$ is the sample thickness (around 120 $\mu \mathrm{m})$ and $L$ is the distance between the two bending points (Figure 2a). The electrical resistance variation due to the mechanical deformation applied to the sensor is represented by the gauge factor $(G F)$, according to equation 2 .

$$
G F=\frac{\Delta R / R_{0}}{\varepsilon}
$$

where $\Delta \mathrm{R}$ is the resistance variation, $R_{0}$ is the initial resistance, and $\varepsilon$ is the applied strain. The piezoresistive effect is the sum of the intrinsic and geometrical piezoresistive effects 46. The GF was calculated for each mechanical stress-strain (load-unload) cycle.

In order to evaluate the viability of the use of the OTFT as a switch in the sensor array, the OTFT and piezoresistive sensor characterization were first performed independently. Then, the electromechanical tests were carried out with the OTFT in its OFF and ON state, thus evaluating the response of the sensor, in particular with the OTFT operating in the zone of saturation and with the highest possible conductivity. 


\section{Results and Discussion}

\section{a) Device geometry}

Figure 5 a shows the printed $5 \times 5$ sensor matrix with a central dimension of $50 \times 50 \mathrm{~mm}^{2}$ (sensor zone) and a total size of $80 \times 65 \mathrm{~mm}^{2}$ (including the terminal of the sensors). It is important to mention that these dimensions can be further reduced, if needed ${ }^{14}$, according to the application and the scalability of production process. A large number of sensors per unit of area can be imperative for high-accuracy applications, but increases the complexity of the matrix.

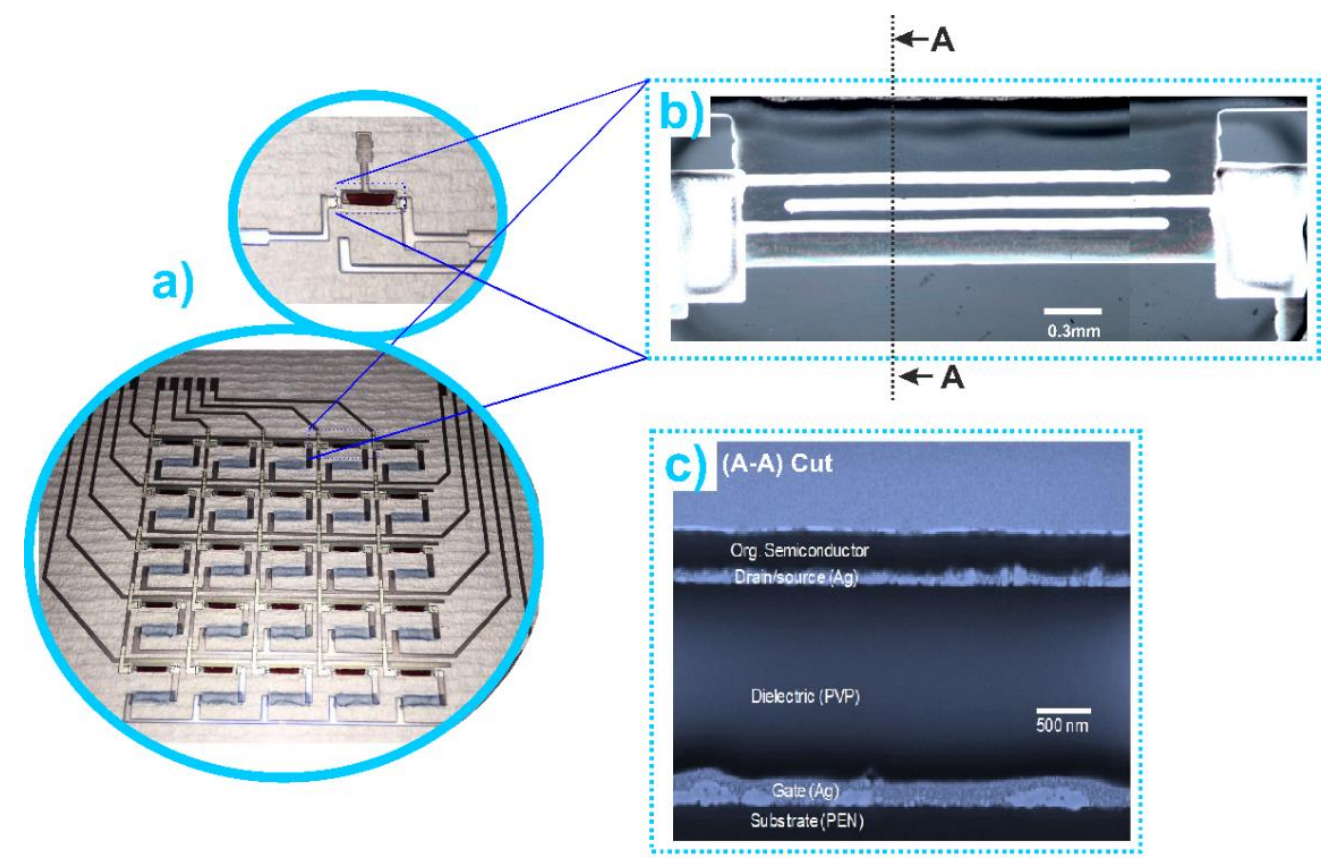

Figure 4. a) Printed piezoresistive sensor matrix, and detail of a single sensor. b) S-D channel detail of the OTFTs, c) cross-sectional profile of the OTFT layer stack.

Figure 4a shows the perfect match between the patterns developed and the final result of the printing, as also verified in the detailed Figure $4 \mathrm{~b}$. 
The structural analysis of the various layers was performed by scanning electron microscopy (SEM) (Figure 4b) and in transversal images obtained by partial removal of material using gallium based focused ion beam (FIB), which also demonstrates the uniformity and proper interface and structure of the different printed layers (Figure 3a). Figure $4 \mathrm{c}$, corresponding to the cross-sectional profile of the various layers, shows that the thickness of the first silver-based conductor layer is about $200 \pm 40 \mathrm{~nm}$, the dielectric layer shows a thickness of $1500 \pm 40 \mathrm{~nm}$, and the second conducting layer, corresponding to the S-D electrodes is characterized by a thickness of about $100 \pm 30 \mathrm{~nm}$. The latter reduction of the thickness relative to the first layer is attributed to the interaction of the printed layer with the previously printed layers as well as to the influence of the printing of the top layers due to the different ink formulations and used solvents. Finally, the semiconductor layer thickness was found about $300 \pm 40 \mathrm{~nm}$. Surface analysis of the printed OTFT, Figure $4 \mathrm{~b}$ ), shows an effective channel width of $\approx 65 \mu \mathrm{m}$ with a channel length of $10.4 \mathrm{~mm}$ and thus a W/L ratio of 130.

\section{b) Electrical characterization of the OTFTs}

The electrical characterization of the OTFTs was performed by measuring the I-V output characteristic curve of the device according to the schematic representation shown in Figure 3c. The I-V response to four VGS is shown in Figure 5a. 

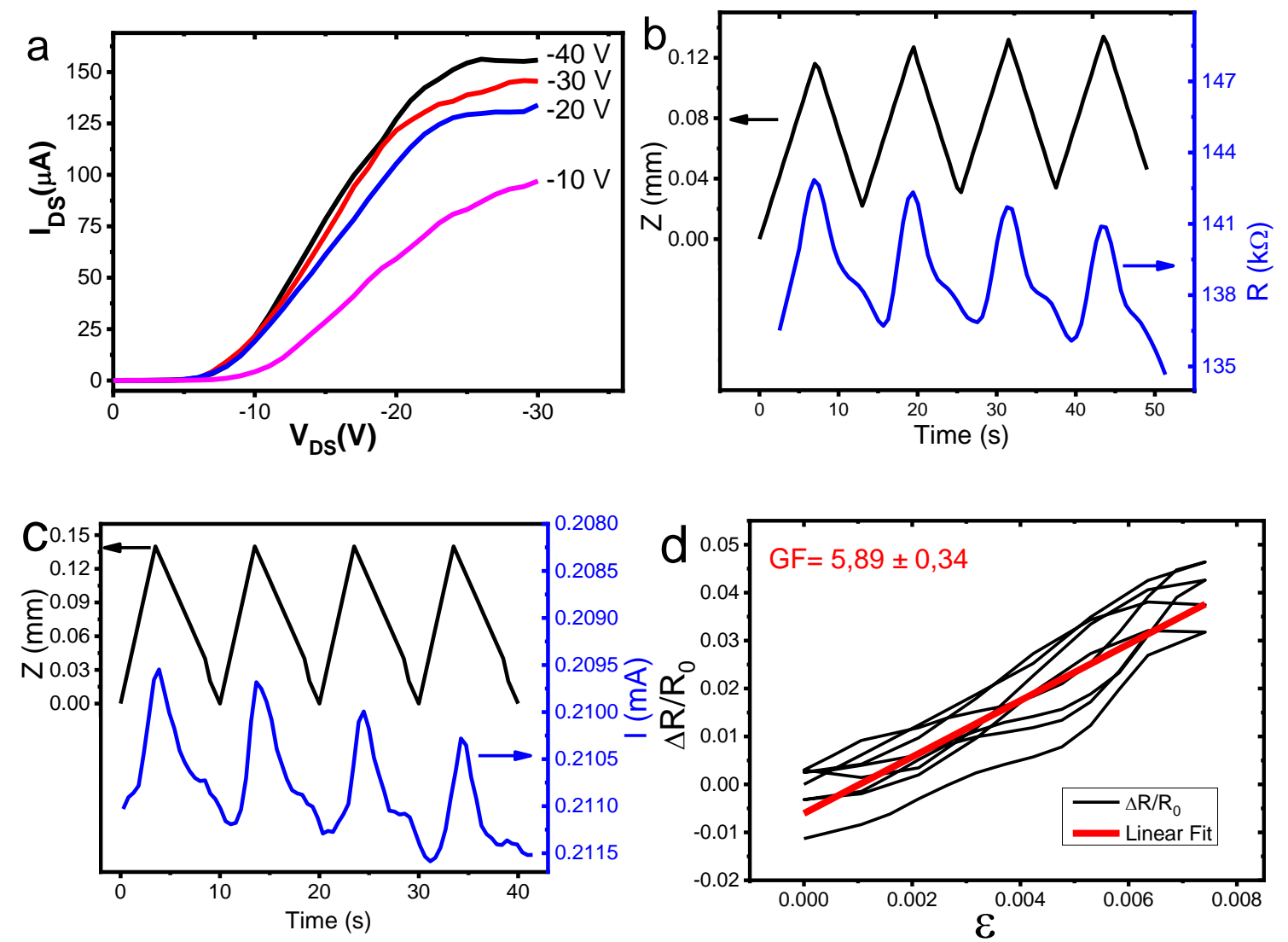

Figure 5. I-V output characteristics of the OTFTs a). Typical individual piezoresistive response of the sensors in the-point bending tests for deformation up to $0.15 \mathrm{~mm}, \mathrm{~b}$ ). Relative resistance variation as function of the applied deformation variation for applied cycles and GF calculation. c) Typical response of the sensors connected to the OTFT in the conduction mode with VGS $=-40 \mathrm{~V}$ and VDS $=-30 \mathrm{~V} \mathrm{~d}$ ).

It was verified that the IDS of the devices increase with increasing applied VDS. On the other hand, IDS saturate with increasing VDS and shows a trend to saturation, following the typical behavior of OTFTs.

The obtained characteristics output curves are similar for all OTFT and typical for transistors based on the material P3HT: $\mathrm{PCBM}^{47,48}$. In this way, a maximum IDS of 150 $\mu \mathrm{A}$ for a VGS of $-40 \mathrm{~V}$ was found. 
To get a reliable comparison of device performance, the transfer characteristics are analyzed from the $\mathrm{ON}$-state to OFF-state (i.e. from VGS $=-40$ to $0 \mathrm{~V}$ ), where the device shows excellent gate modulation with a current on/off ratio of more than 105 .

In general, it is possible to improve the performance of the OTFT, e.g. by reducing the thickness of the dielectric layer, ensuring the non-electrical breakdown of the layer, or through geometrical variation of the channel (i.e., increase of the length and/or decrease of the width) or by the usage of other materials.

\section{c) Piezoresistive response}

First, the reproducibility of the sensor's manufacturing method was evaluated by measuring $\mathrm{R}_{0}$ for the different sensors within the matrix. Good reproducibility is verified due to a variation of $\mathrm{R}_{0}$ in the range of $135 \mathrm{k} \Omega$ and $165 \mathrm{k} \Omega$ (average resistance about 150 $\mathrm{k} \Omega$, in agreement with the applied manufacturing process and conditions) ${ }^{37}$.

With respect to the piezoresistive response, Figure $5 \mathrm{~b}$ shows the typical individual response of the piezoresistive sensors to a mechanical solicitation. Where it is verified that the resistance variation is of the order of $3 \%$, linearly, for a complete mechanical deformation cycle. This response is related to the small size of the sensor of $8 \times 2 \mathrm{~mm}^{2}$.

On the other hand, there is also a small attenuation of the response over the several cycles due to the total non-recovery of the material motivated by the viscoelastic mechanical characteristics of the polymer. This is leading to a slow mechanical relaxation of the applied strain and thus to slower reconfiguration of the MWCNT network which results in the related electrical response ${ }^{35}$. This effect diminishes with increasing the number of cycles to which the material is subjected as demonstrated $\mathrm{in}^{35}$. 
Analyzing the variation of the resistance as a function of the applied deformation, it is possible to calculate the $G F$ for the various applied cycles based on equation 2 (Figure 5 c). The average $G F$ for the sensors is $5.9 \pm 0.3$.

Finally, the sensors were measured in conjunction with the excitation of the OTFTs, i.e. placing the OTFTs in the conduction state, applying a voltage of VGS of $-40 \mathrm{~V}$, corresponding to the highest conduction voltage of the OTFT, and applying a voltage between the source and end of the sensor of $-30 \mathrm{~V}$ (Figure 6). Thus, the OTFT is placed to operate in the saturation zone, functioning as a switch. In this way, the variation of the current, corresponding to the series between the source-drain resistor RSD and the resistance of the sensor, is evaluated as represented in Figure 5b). Therefore, it is possible to measure each sensor independently, avoiding the cross-talking between them since the resistance of the OTFTs that is not in conduction mode is larger than $100 \mathrm{M} \Omega$, whereas the resistance in the conduction mode is about 400 times smaller (approximately 0.25 $\mathrm{M} \Omega$ ).

Therefore, any variation in the sensors with the corresponding OTFT in the OFF state will be lower when compared to the sensor response when the OTFT is in ON state. In this way, it is possible to measure the response of the different sensors within the matrix independently, eliminating the crosstalk between them.

There is an attenuation in the sensor response to a deformation cycle from a variation of $3 \%$ just for the sensor to a variation of $0.7 \%$ for the sensor with the OTFT. This phenomenon is due to the fact, that resistance of approximately $0.2 \mathrm{M} \Omega$ is placed in series with the resistance of the sensor. A compensation by amplification with a signal acquisition circuit can be applied easily.

Alternatively, this attenuation can be also minimized by improving the characteristics of the transistor by reducing its $\mathrm{R}_{\mathrm{ON}}$ or by increasing the $\mathrm{R}_{0}$ value of the sensor to be as large 
as possible when compared to the $\mathrm{R}_{\mathrm{ON}}$ of the transistor. The latter solution, on the other hand, will lead to a decrease of the measured current, which can result in an increase in the noise.

\section{Conclusions}

The present contribution demonstrates a fully printed sensor matrix on a flexible polymer substrate suitable for a large range of applications. A reduction of crosstalk between sensors due to the implementation of OTFTs to the sensing piezoresistive layers was obtained (about 150 times reduced compared to a sensor without OTFT). The piezoresistive sensors had a $G F \approx 5.9$.

The entire sensor matrix and the OTFTs were fabricated by inkjet and screen-printing technologies, demonstrating the high potential of this technology as a suitable manufacturing tool for sensors. The OTFTs were fabricated with three different types of ink formulations: silver nanoparticle ink (conductive ink for the electrodes), polymeric cPVP ink (dielectric ink formulation) and P3HT:PCBM (as semiconducting ink).

The performance of the OTFTs was studied in detail. A S-D current of $150 \mu \mathrm{A}$ for a G$\mathrm{S}$ voltage of $-40 \mathrm{~V}$ with a W/L of 130 was obtained. In order to finalize the sensor device, screen printing was applied for the deposition of the piezoresistive layer on top of the inkjet-printed matrix. The piezoresistive layer is based on SEBS and MWCNT.

The introduced concept of the printed sensor matrix can be also extended to other types of resistive sensors, where the crosstalk can hinder the interpretation of the obtained results. In addition, the printing technologies could be used for the fabrication of other types of sensors as well.

\section{AUTHOR INFORMATION}




\section{Corresponding Authors}

Pedro Costa: pcosta@fisica.uminho.pt

S. Lanceros-Mendez: senentxu.lanceros@bcmaterials.net

\section{Author Contributions}

The manuscript was written through contributions of all authors. All authors have given approval to the final version of the manuscript.

\section{Funding Sources}

FCT - Portuguese Foundation for Science and Technology

Basque Government Industry Department

\section{ACKNOWLEDGMENTS}

This work has been supported by national funds through FCT - Fundação para a Ciência e Tecnologia within the Project Scope: UID/CEC/00319/2019, UID/FIS/04650/2019 and projects PTDC/FIS-MAC/28157/2017 and PTDC/BTM-MAT/28237/2017, SFRH/BPD/110914/2015 (PC). V.C. thanks FCT for the junior researcher contract (DL57/2016). We acknowledge funding from the European Union's Horizon 2020 Programme for Research, ICT-02-2018 - Flexible and Wearable Electronics. Grant agreement no. 824339 - WEARPLEX. Financial support from the Basque Government Industry and Education Department under the ELKARTEK, HAZITEK and PIBA (PIBA-2018-06) programs, respectively, is also acknowledged.

\section{REFERENCES}

(1) Vuran, M. C.; Salam, A.; Wong, R.; Irmak, S. Ad Hoc Networks Internet of Underground Things in Precision Agriculture : Architecture and Technology Aspects R. Ad Hoc Networks 2018, 81, 160-173. 
https://doi.org/10.1016/j.adhoc.2018.07.017.

(2) Lou, Z.; Wang, L.; Shen, G. Recent Advances in Smart Wearable Sensing Systems. 2018, 1800444, 1-17. https://doi.org/10.1002/admt.201800444.

(3) Yeom, C.; Chen, K.; Kiriya, D.; Yu, Z.; Cho, G.; Javey, A. Large-Area Compliant Tactile Sensors Using Printed Carbon Nanotube Active-Matrix Backplanes. Adv. Mater. 2015, 27 (9), 1561-1566. https://doi.org/10.1002/adma.201404850.

(4) Clark, J. Self-Calibration and Performance Control of MEMS with Applications for IoT. Sensors (Basel) 2018. https://doi.org/10.3390/s18124411.

(5) Yao, S.; Ren, P.; Song, R.; Liu, Y.; Huang, Q.; Dong, J.; O’Connor, B. T.; Zhu, Y. Nanomaterial-Enabled Flexible and Stretchable Sensing Systems: Processing, Integration, and Applications. Adv. Mater. $n / a$ (n/a), 1902343. https://doi.org/10.1002/adma.201902343.

(6) Duan, Z.; Jiang, Y.; Wang, S.; Yuan, Z.; Zhao, Q.; Xie, G.; Du, X.; Tai, H. Inspiration from Daily Goods: A Low-Cost, Facilely Fabricated, and Environment-Friendly Strain Sensor Based on Common Carbon Ink and Elastic Core-Spun Yarn. ACS Sustain. Chem. Eng. 2019, 7 (20), 17474-17481. https://doi.org/10.1021/acssuschemeng.9b04690.

(7) Duan, Z.; Jiang, Y.; Yan, M.; Wang, S.; Yuan, Z.; Zhao, Q.; Sun, P.; Xie, G.; Du, $\mathrm{X}$; Tai, H. Facile, Flexible, Cost-Saving, and Environment-Friendly Paper-Based Humidity Sensor for Multifunctional Applications. ACS Appl. Mater. Interfaces 2019, 11 (24), 21840-21849. https://doi.org/10.1021/acsami.9b05709.

(8) Duan, Z.; Zhao, Q.; Wang, S.; Yuan, Z.; Zhang, Y.; Li, X.; Wu, Y.; Jiang, Y.; Tai, H. Novel Application of Attapulgite on High Performance and Low-Cost Humidity Sensors. Sensors Actuators B Chem. 2020, 305, 127534. https://doi.org/https://doi.org/10.1016/j.snb.2019.127534.

(9) Seekaew, Y.; Lokavee, S.; Phokharatkul, D.; Wisitsoraat, A. Low-Cost and Flexible Printed Graphene - PEDOT : PSS Gas Sensor for Ammonia Detection. Org. Electron. 2014, 15 (11), 2971-2981. https://doi.org/10.1016/j.orgel.2014.08.044.

(10) Tai, H.; Duan, Z.; He, Z.; Li, X.; Xu, J.; Liu, B.; Jiang, Y. Enhanced Ammonia Response of Ti3C2Tx Nanosheets Supported by TiO2 Nanoparticles at Room Temperature. Sensors Actuators B Chem. 2019, 298, 126874. https://doi.org/https://doi.org/10.1016/j.snb.2019.126874.

(11) Amadeo, M.; Campolo, C.; Molinaro, A.; Ruggeri, G. IoT Data Processing at the Edge with Named Data Networking. Eur. Wirel. 2018 2018, 38-43.

(12) Yong, N.; Jung, K.; Ryu, H.; Wook, B.; Yi, K.; Park, J. H. CF-CloudOrch : Container Fog Node-Based Cloud Orchestration for IoT Networks. $J$.

Supercomput. 2018, 74 (12), 7024-7045. https://doi.org/10.1007/s11227-0182493-4.

(13) Abdelbadie, S. A.; Mikhael, A. A.; Helmy, M. M.; Elgharabawy, B. A.; Mohieldin, A. N. An Ultra-Low-Power RF Receiver for IoT Applications Using 65nm CMOS Technology. 2018 7th Int. Conf. Mod. Circuits Syst. Technol. 2018, 1-5. https://doi.org/10.1109/MOCAST.2018.8376581.

(14) Oliveira, J.; Correia, V.; Castro, H.; Martins, P.; Lanceros-mendez, S. PolymerBased Smart Materials by Printing Technologies : Improving Application and Integration. Addit. Manuf. 2018, 21 (October 2017), 269-283. https://doi.org/10.1016/j.addma.2018.03.012.

(15) Semiconductor, O. $150 \mathrm{~mA}$, Ultra Low Supply Current, Low Dropout Regulator http://www.onsemi.com/pub_link/Collateral/NCP4682-D.PDF. 
(16) Sowade, E.; Ramo, E.; Mitra, K. Y.; Martínez-domingo, C.; Pedró, M.; Pallarès, J.; Loffredo, F.; Villani, F.; Gomes, H. L.; Terés, L.; Baumann, R. R.

Transistors : Manufacturing Process Reliability by Root Cause Analysis. Sci. Rep. 2016, No. August, 1-15. https://doi.org/10.1038/srep33490.

(17) Mraovi, M.; Bra, S.; Muck, D. Printed Thermochromic Displays Coloration Technology. Color. Technol. 2018, 60-66. https://doi.org/10.1111/cote.12372.

(18) Moya, A.; Sowade, E.; del Campo, F. J.; Mitra, K. Y.; Ramon, E.; Villa, R.; Baumann, R. R.; Gabriel, G. All-Inkjet-Printed Dissolved Oxygen Sensors on Flexible Plastic Substrates. Org. Electron. 2016, 39, 168-176. https://doi.org/https://doi.org/10.1016/j.orgel.2016.10.002.

(19) Costa, P.; Ferreira, A.; Sencadas, V.; Viana, J. C.; Lanceros-méndez, S. Physical Electro-Mechanical Properties of Triblock Copolymer Styrene - Butadiene Styrene / Carbon Nanotube Composites for Large Deformation Sensor Applications. Sensors Actuators A. Phys. 2013, 201, 458-467. https://doi.org/10.1016/j.sna.2013.08.007.

(20) Oliveira, J.; Correia, V.; Sowade, E.; Etxebarria, I.; Rodriguez, R. D.; Mitra, K. Y.; Baumann, R. R.; Lanceros-Mendez, S. Indirect X-Ray Detectors Based on Inkjet-Printed Photodetectors with a Screen-Printed Scintillator Layer. ACS Appl. Mater. Interfaces 2018, 10 (15), 12904-12912. https://doi.org/10.1021/acsami.8b00828.

(21) Castro, H. F.; Correia, V.; Sowade, E.; Mitra, K. Y.; Rocha, J. G.; Baumann, R. R.; Lanceros-M??ndez, S. All-Inkjet-Printed Low-Pass Filters with Adjustable Cutoff Frequency Consisting of Resistors, Inductors and Transistors for Sensor Applications. Org. Electron. physics, Mater. Appl. 2016, 38. https://doi.org/10.1016/j.orgel.2016.08.025.

(22) Correia, V.; Mitra, K. Y.; Castro, H.; Rocha, J. G.; Sowade, E.; Baumann, R. R.; Lanceros-Mendez, S. Design and Fabrication of Multilayer Inkjet-Printed Passive Components for Printed Electronics Circuit Development. J. Manuf. Process. 2018, 31, 364-371. https://doi.org/https://doi.org/10.1016/j.jmapro.2017.11.016.

(23) Sowade, E.; Polomoshnov, M.; Willert, A.; Baumann, R. R. Toward 3D-Printed Electronics : Inkjet-Printed Vertical Metal Wire Interconnects and Screen-Printed Batteries. Adv. Eng. Mater. 2019, 1900568, 1-8. https://doi.org/10.1002/adem.201900568.

(24) Lanceros-Méndez, S.; Costa, C. M. Printed Batteries: Materials, Technologies and Applications; Wiley, 2018.

(25) Lee, W.; Koo, H.; Sun, J.; Noh, J.; Kwon, K.-S.; Yeom, C.; Choi, Y.; Chen, K.; Javey, A.; Cho, G. A Fully Roll-to-Roll Gravure-Printed Carbon Nanotube-Based Active Matrix for Multi-Touch Sensors. Sci. Rep. 2015, 5 (1), 17707. https://doi.org/10.1038/srep17707.

(26) Fiorillo, A. S.; Critello, C. D.; Pullano, S. A. Physical Theory, Technology and Applications of Piezoresistive Sensors : A Review. Sensors Actuators A. Phys. 2018, 281, 156-175. https://doi.org/10.1016/j.sna.2018.07.006.

(27) Adhikari, B.; Majumdar, S. Polymers in Sensor Applications. Prog. Polym. Sci. 2004, 29, 699-766. https://doi.org/10.1016/j.progpolymsci.2004.03.002.

(28) Fink, J. K. Polymeric Sensors and Actuators; Polymer Science and Plastics Engineering; Wiley, 2012.

(29) Wang, Y.; Tian, Y.; Yu, X. Detection of Abrin Using a Polymide-Based Piezoresistive Microcantilever Biosensor. 2018 14th IEEE Int. Conf. Solid-State Integr. Circuit Technol. 2018, 1-3.

(30) Wagner, S.; Yim, C.; McEvoy, N.; Kataria, S.; Yokaribas, V.; Kuc, A.; Pindl, S.; 
Fritzen, C.-P.; Heine, T.; Duesberg, G. S.; Lemme, M. C. Highly Sensitive Electromechanical Piezoresistive Pressure Sensors Based on Large-Area Layered PtSe2 Films. Nano Lett. 2018, 18 (6), 3738-3745.

https://doi.org/10.1021/acs.nanolett.8b00928.

(31) Alpuim, P.; Correia, V.; Marins, E. S.; Rocha, J. G.; Trindade, I. G.; LancerosMendez, S. Piezoresistive Silicon Thin Film Sensor Array for Biomedical Applications. Thin Solid Films 2011, 519 (14), 4574-4577. https://doi.org/10.1016/j.tsf.2011.01.300.

(32) Cui, J.; Zhang, B.; Duan, J.; Guo, H.; Tang, J. Flexible Pressure Sensor with Ag Wrinkled Electrodes Based on PDMS Substrate. Sensors 2016. https://doi.org/10.3390/s16122131.

(33) Tires, P. Flexible Piezoresistive Sensors Embedded in 3D Printed Tires. 2017. https://doi.org/10.3390/s17030656.

(34) Gonçalves, B. F.; Costa, P.; Oliveira, J.; Ribeiro, S.; Correia, V.; Botelho, G.; Lanceros-Mendez, S. Green Solvent Approach for Printable Large Deformation Thermoplastic Elastomer Based Piezoresistive Sensors and Their Suitability for Biomedical Applications. J. Polym. Sci. Part B Polym. Phys. 2016, 54 (20), 2092-2103. https://doi.org/10.1002/polb.24118.

(35) Costa, P.; Oliveira, J.; Horta-romarís, L.; Abad, M.; Moreira, J. A.; Zapiráin, I.; Aguado, M.; Galván, S.; Lanceros-mendez, S. Piezoresistive Polymer Blends for Electromechanical Sensor Applications. Compos. Sci. Technol. 2018, 168 (September), 353-362. https://doi.org/10.1016/j.compscitech.2018.10.022.

(36) Castro, H. F.; Correia, V.; Pereira, N.; Costab, P.; Oliveiraa, J.; LancerosMéndez, S. Printed Wheatstone Bridge with Embedded Polymer Based Piezoresistive Sensors for Strain Sensing Applications. Addit. Manuf. 2018, 20, 119-125. https://doi.org/https://doi.org/10.1016/j.addma.2018.01.004.

(37) Tebyetekerwa, M.; Wang, X.; Marriam, I.; Dan, P.; Yang, S.; Zhu, M. Green Approach to Fabricate Polyindole Composite Nanofibers for Energy and Sensor Applications. Mater. Lett. 2017, 209, 400-403. https://doi.org/10.1016/j.matlet.2017.08.062.

(38) Mantysalo, M.; Mansikkamaki, P.; Miettinen, J.; Kaija, K.; Pienimaa, S.; Ronkka, R.; Hashizume, K.; Kamigori, A.; Matsuba, Y.; Oyama, K.; Terada, N.; Saito, H.; Kuchiki, M.; Tsubouchi, M. Evaluation of Inkjet Technology for Electronic Packaging and System Integration. Electronic Components and Technology Conference, 2007. ECTC '07. Proceedings. 57th. 2007, pp 89-94. https://doi.org/10.1109/ECTC.2007.373781.

(39) Liao, Z.; Wang, J.; Zhang, P.; Zhang, Y.; Miao, Y.; Gao, S. Biosensors and Bioelectronics Recent Advances in Micro Fl Uidic Chip Integrated Electronic Biosensors for Multiplexed Detection. Biosens. Bioelectron. 2018, 121 (June), 272-280. https://doi.org/10.1016/j.bios.2018.08.061.

(40) Choi, H. W.; Zhou, T.; Jabbour, G. E. Recent Developments and Directions in Printed Nanomaterials. Nanoscale 2014, 7, 3338-3355. https://doi.org/10.1039/c4nr03915g.

(41) Z.P., Y.; Y.A., H.; N.B., B.; X.M., W.; Y.L., X. Inkjet Printing for Flexible Electronics: Materials, Processes and Equipments. Chinese Sci. Bull. 2010, 55 (30), 3383-3407. https://doi.org/10.1007/s11434-010-3251-y.

(42) Correia, V.; Caparros, C.; Casellas, C.; Francesch, L.; Rocha, J. G.; LancerosMendez, S. Development of Inkjet Printed Strain Sensors. Smart Mater. Struct. 2013, 22 (10). https://doi.org/10.1088/0964-1726/22/10/105028.

(43) Gonçalves, B. F.; Costa, P.; Oliveira, J.; Ribeiro, S.; Correia, V.; Botelho, G.; 
Lanceros-Mendez, S. Green Solvent Approach for Printable Large Deformation Thermoplastic Elastomer Based Piezoresistive Sensors and Their Suitability for Biomedical Applications. J. Polym. Sci. Part B Polym. Phys. 2016, 54 (20), 2092-2103. https://doi.org/10.1002/polb.24118.

(44) Lorwongtragool, P.; Sowade, E.; Watthanawisuth, N.; Baumann, R. R.;

Kerdcharoen, T. A Novel Wearable Electronic Nose for Healthcare Based on Flexible Printed Chemical Sensor Array. Sensors 2014, 14 (10), 19700-19712. https://doi.org/10.3390/s141019700.

(45) Gonçalves, B. F.; Oliveira, J.; Costa, P.; Correia, V.; Martins, P.; Botelho, G.; Lanceros-Mendez, S. Development of Water-Based Printable Piezoresistive Sensors for Large Strain Applications. Compos. Part B Eng. 2017, 112, 344-352. https://doi.org/https://doi.org/10.1016/j.compositesb.2016.12.047.

(46) Correia, V.; Caparros, C.; Casellas, C.; Francesch, L.; Rocha, J. G.; LancerosMendez, S. Development of Inkjet Printed Strain Sensors. J. Appl. Mater. Interfaces 2013, Submitted.

(47) Pal, T.; Arif, M.; Khondaker, S. I. High Performance Organic Phototransistor Based on Regioregular Poly ( 3-Hexylthiophene ). Nanotechnology 2010. https://doi.org/10.1088/0957-4484/21/32/325201.

(48) Kumar, C.; Rawat, G.; Kumar, H.; Kumar, Y.; Prakash, R.; Jit, S. Electrical and Ammonia Gas Sensing Properties of Poly ( 3, 3000 - Dialkylquaterthiophene ) Based Organic Thin Fi Lm Transistors Fabricated by Fl Oating- Fi Lm Transfer Method. Org. Electron. 2017, 48, 53-60. https://doi.org/10.1016/j.orgel.2017.05.040.

\section{TOC}

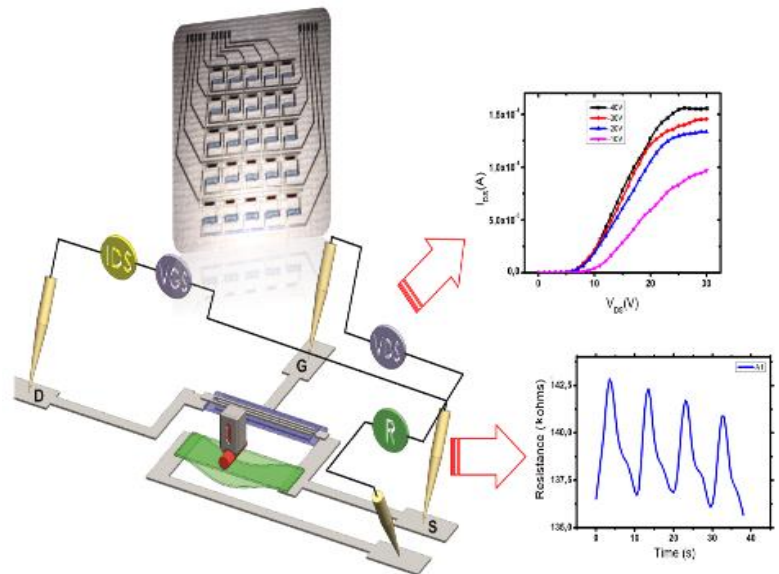

